\title{
TFIID and human mediator coactivator complexes assemble cooperatively on promoter DNA
}

\author{
Kristina M. Johnson, Jin Wang, ${ }^{1}$ Andrea Smallwood, Charina Arayata, and Michael Carey ${ }^{2}$ \\ Department of Biological Chemistry, University of California, Los Angeles School of Medicine, \\ Los Angeles, California 90095-1737, USA
}

\begin{abstract}
Activator-mediated transcription complex assembly on templates lacking chromatin requires the interaction of activators with two major coactivator complexes: TFIID and mediator. Here we employed immobilized template assays to correlate transcriptional activation with mediator and TFIID recruitment. In reactions reconstituted with purified proteins, we found that activator, TFIID, and mediator engage in reciprocal cooperative interactions to form a complex on promoter DNA. Preassembly of the coactivator complex accelerates the rate of transcription in a cell-free system depleted of TFIID and mediator. Our data argue that this coactivator complex is an intermediate in the assembly of an active transcription complex. Furthermore, the reciprocity of the interactions demonstrates that the complex could in principle be nucleated with either TFIID or mediator, implying that alternative pathways could be utilized to generate diversity in the way activators function in vivo.
\end{abstract}

[Key Words: TFIID; mediator; GAL4-VP16; activation; cooperativity]

Received April 1, 2002; revised version accepted May 22, 2002.

Highly regulated signaling pathways and chromatin environments have evolved to modulate the activity of gene activators and to control their access to promoter DNA. A fundamental question is: How do activators stimulate high levels of transcription by RNA polymerase II after chromatin remodeling is complete? The current model is that activators function through recruitment of the general transcription machinery (Ptashne and Gann 1997). The general machinery (Woychik and Hampsey 2002) comprises the general transcription factors (GTFs) required for basal transcription (TFIIA, TFIIB, TBP, TFIIE, TFIIF, TFIIH) and two coactivator complexes: TFIID, with its TBP-associated factors (TAFs) (Hahn 1998), and the mediator complex (Myers et al. 1999; Malik and Roeder 2000).

Initial studies of transcription complex assembly in fractionated human systems indicated that binding of TFIID to the TATA box nucleates assembly of the preinitiation complex (PIC) (Buratowski et al. 1989; Van Dyke et al. 1989). Incubation of template and crude TFIID and TFIIA fractions (the DA complex) with activators overcomes rate-limiting steps in transcription

\footnotetext{
${ }^{1}$ Present address: Department of Biochemistry, Nanjing University, Nanjing, People's Republic of China.

${ }^{2}$ Corresponding author.

E-MAIL mcarey@ucla.edu; FAX (310) 206-9598.

Article and publication are at http://www.genesdev.org/cgi/doi/10.1101/ gad.995702.
}

complex assembly (Wang et al. 1992b). Indeed, numerous activators from USF to GAL4-VP16 recruit DA or stabilize its binding in vitro (Sawadogo and Roeder 1985; Horikoshi et al. 1988a,b; Lieberman and Berk 1994; Chi et al. 1995). Interaction of the DA complex with activators generates a conformational change, which is both necessary and sufficient for gene activation (Horikoshi et al. 1988b; Chi and Carey 1996; Shykind et al. 1997).

Purified TFIID, however, fails to support robust activated transcription when incubated with the remaining GTFs (discussed in Baek et al. 2002). A fraction termed USA was found to contain coactivators that permit robust, activated transcription (Meisterernst et al. 1991). Subsequent studies showed that the USA fraction contains a large, multisubunit assembly termed PC2 and several other positive and negative cofactors (Kretzschmar et al. 1994). PC2 is a subcomplex of the human mediator (Malik et al. 2000).

The mediator coactivator complex was first purified from yeast as an activity that relieved squelching by the strong, model activator, GAL4-VP16 (Kelleher et al. 1990; Flanagan et al. 1991; Myers and Kornberg 2000). Subunits of the same complex were also identified by a genetic screen for suppressors of mutations in the carboxyl terminal domain (CTD) of the largest subunit of RNA polymerase II (Thompson et al. 1993). The yeast mediator is capable of supporting activated transcription when supplemented with purified general transcription factors and PolII (Kim et al. 1994; Myers and Kornberg 
2000). This discovery led to the model that activators interact with the mediator complex, which then mediates communication with the GTFs and PolII (Myers and Kornberg 2000).

The existence of a human equivalent of the yeast mediator (hMed) was indicated by the USA fraction (Meisterernst et al. 1991) and by later studies of thyroid receptor-associated proteins or TRAPs (Fondell et al. 1999). Human mediator was eventually purified by several groups, who utilized different strategies for its isolation (for review, see Malik and Roeder 2000). The complex has been variously termed TRAP (Fondell et al. 1999), SMCC (Gu et al. 1999), NAT (Sun et al. 1998), ARC (Naar et al. 1999), DRIP (Rachez et al. 1999), CRSP (Ryu et al. 1999), human mediator (Boyer et al. 1999), and PC2 of USA (Malik et al. 2000). In this article we will use the Med subunit nomenclature proposed by Rachez and Freedman (2001). Cloning and analysis of hMed subunits revealed some minor but noteworthy homologies to yeast mediator counterparts (for review, see Malik and Roeder 2000).

Human mediator interacts directly with activators and undergoes specific conformational changes (Taatjes et al. 2002). Recent reports indicate that two distinct forms of the mediator complex exist in mammalian cells, of which only one is highly active in vitro (Taatjes et al. 2002). The current view, based on work in yeast, where deletion of a core subunit of yeast mediator, SRB4, causes global defects in transcription (Holstege et al. 1998), is that hMed is universally required for transcriptional activation. It is believed that individual activators can independently contact various mediator subunits (Ito et al. 1999).

Over a decade of work has provided strong evidence of the critical nature of both TFIID and mediator in activated transcription (Walker et al. 1996; Holstege et al. 1998). Activators have been shown to interact directly with subunits of either or, in some cases, both the TFIID and mediator complexes in yeast and human (Hahn 1998; Malik and Roeder 2000). The mediator was shown to influence basal transcription (Mittler et al. 2001; Baek et al. 2002), suggesting that it may contact GTFs directly in a physiological context. Further, mediator and TFIID act synergistically to support activated transcription in a reaction requiring TAFs (Guermah et al. 2001; Baek et al. 2002). In this paper we propose that activators recruit both of these coactivator complexes simultaneously via formation of a higher-order assembly. Cooperative interactions between activators, TFIID, and hMed are critical for formation of this higher-order complex, which stimulates PIC assembly and thus plays an essential role in regulated transcription.

The existence of an important, functional interaction between TFIID and mediator is supported by a study in yeast. This study indicated that the mediator and TFIID complexes, along with substoichiometric amounts of two GTFs, are bound together at a promoter in a reinitiation scaffold, whose stability is dependent on the strength of an activator (Yudkovsky et al. 2000). Although its relevance to initiation was not demonstrated, this was the first indication that a stable interaction might exist between these two coactivator complexesan observation critical to the argument for cooperative coactivator assembly.

The present study was designed to address whether purified TFIID and hMed coactivator complexes can cooperatively assemble on promoter DNA in a functionally relevant manner. We used the model activator GAL4VP16 (Carey et al. 1990a) and an immobilized template system to study coactivator recruitment. Purified hMed is recruited to immobilized templates by GAL4-VP16 and, in turn, enhances recruitment of purified TFIID. Conversely, TFIID stimulates recruitment of hMed to promoter DNA. We present evidence that under conditions of cooperative recruitment of hMed and TFIID, preassembly of this higher-order coactivator complex overcomes a rate-determining step in activated transcription.

\section{Results}

GAL4-VP16 stimulates recruitment of both the human mediator complex and TFIID from nuclear extract to promoter DNA

Our study was designed to test the hypothesis that activated transcription involves cooperative recruitment of TFIID and hMed. To correlate transcriptional activity with recruitment, we employed an immobilized template system (Kim et al. 1998; Ranish et al. 1999; Yie et al. 1999). The promoter we used contains five GAL4 DNA binding sites upstream of the adenovirus E4 TATA box: $\mathrm{G}_{5}$ E4T (Fig. 1A; Carey et al. 1990a). Linearized, biotinylated templates were immobilized via fusion to streptavidin-coated magnetic beads (Dynabeads ${ }^{\mathrm{TM}}$ ). These immobilized DNA fragments were used as templates for transcription and, in parallel, were used to isolate, visualize, and quantitate by immunoblotting components of the PIC bound to the template.

GAL4-VP16 binds to the immobilized template in amounts proportional to the number of GAL4 binding sites on the DNA (Fig. 1B). GAL4-VP16 that is bound to an immobilized template bearing five GAL4 DNA binding sites can stimulate transcription in HeLa nuclear extract (NE) (Fig. 1C).

Parallel binding and transcription experiments show that recruitment of the transcription machinery (PIC assembly) by GAL4-VP16 correlates with transcriptional activity. Figure 1D illustrates that binding of GAL4VP16 correlates with enhanced binding of all components of the transcription machinery tested (Fig. D, cf. lanes 1 and 2). Note the increased presence of hMed, as represented by the subunits Med220/TRAP220/ DRIP205/ARC205 (Yuan et al. 1998), Med130/hSUR2/ TRAP150/DRIP130/ARC130 (Boyer et al. 1999), and Med33/hMed6/DRIP33/ARC33 (Gu et al. 1999), and the stimulation of TFIID recruitment, assayed by the presence of TBP and $\mathrm{TAF}_{\mathrm{II}} 250$ (Hisatake et al. 1993; Ruppert et al. 1993). To study the potential cooperative recruitment of the TFIID and human mediator coactivators, we 
Johnson et al.

A

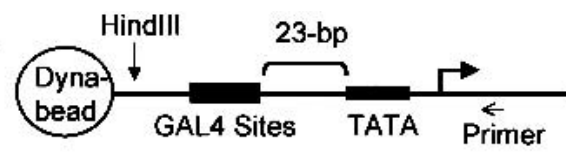

B GAL4 Sites: 0125

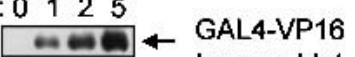

Immunoblot

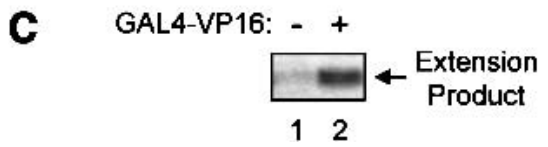

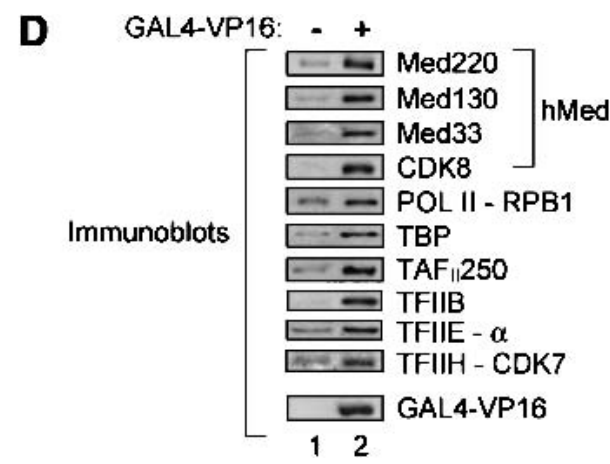

Figure 1. Transcriptional activation by GAL4-VP16 correlates with recruitment of the general machinery. (A) Schematic of the 650-bp immobilized template bearing five GAL4 DNA binding sites $23 \mathrm{bp}$ upstream of the adenovirus E4 TATA box. The HindIII site is used to cleave the DNA from the Dynabeads ${ }^{\mathrm{TM}}$ for quantitation, by agarose gel electrophoresis. $(B)$ The amount of GAL4-VP16 protein bound to the immobilized templates is proportional to the number of GAL4 DNA binding sites. Two hundred ng purified, recombinant GAL4-VP16 was added to $25 \mathrm{fmol}$ immobilized template bearing 0, 1, 2, or 5 GAL4 DNA binding sites. The bound protein was fractionated by SDS-PAGE. An immunoblot of GAL4-VP16 is shown. (C) GAL4-VP16 stimulates activated levels of transcription from the immobilized templates. One hundred-twenty micrograms of HeLa nuclear extract was added in batch with 5 ng GAL4-VP16 and $10 \mathrm{fmol}$ immobilized DNA template. After $1 \mathrm{~h}$ at $30^{\circ} \mathrm{C}$, the transcription products were analyzed by primer extension analysis (arrows denote extension product) and resolved on a $10 \%$ polyacrylamide/urea gel. A phosphorimager scan of the gel is shown. (D) GAL4-VP16 stimulates recruitment of general transcription factors and the human mediator coactivator complex to immobilized templates. One hundred-twenty micrograms of HeLa nuclear extract was incubated with $10 \mathrm{fmol}$ immobilized DNA template in the absence (lane 1) or presence (lane 2) of $5 \mathrm{ng}$ GAL4-VP16. After $1 \mathrm{~h}$ at room temperature, the beads were washed and bound protein was fractionated on $4 \%-15 \%$ SDS polyacrylamide gels. Individual factors were assayed by immunoblot. Autoradiographs are shown from several experiments measuring different sets of factors.

purified active complexes and subjected these to immobilized template recruitment analysis.

\section{Composition and activity of purified human mediator}

The human mediator coactivator complex (hMed) was isolated using two published affinity purification approaches (Rachez et al. 1998; Naar et al. 1999). First, we employed an affinity method initially described by Freedman and colleagues, which utilizes a GST-TR $\mathrm{LBD}_{\mathrm{D}}$ fusion matrix (Rachez et al. 1998). Figure 2A, lanes 3-5 illustrate, in part, the composition of the purified fraction. As has been reported (Gu et al. 1999), we observe copurification of RNA polymerase II with hMed. We surmise based on work from the Roeder group, and our analysis of silver-stained gels of our hMed preparation,

Figure 2. Composition and activity of A purified human mediator (hMed) and mediator-depleted nuclear extract $(\Delta \mathrm{NE})$. (A) HeLa nuclear extracts were fractionated on a GST-TR $\mathrm{LBD}_{\mathrm{L}}$ affinity matrix. The starting (lane 1) and depleted extract (lane 2) along with increasing amounts of mediator fraction (lanes 3-5) were fractionated on $4 \%-15 \%$ SDS polyacrylamide gels and analyzed by immunoblot with antibodies against representative subunits of hMed and GTFs. Autoradiographs of the blots are shown. Note that hMed is depleted from the $\Delta$ NE. Med220 is depleted greater than 30 -fold. Also, general transcription factors, including TBP and TAFs of TFIID, are present in the $\triangle \mathrm{NE}$ fraction but are not present in

NE ANE hMed
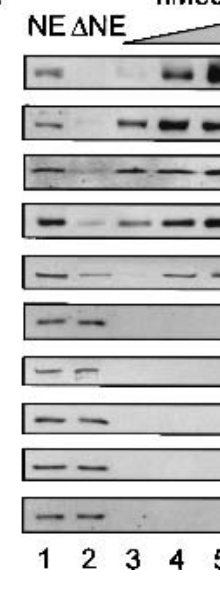

B

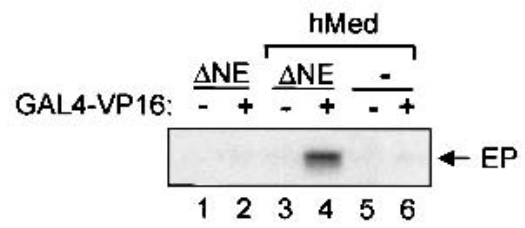

C

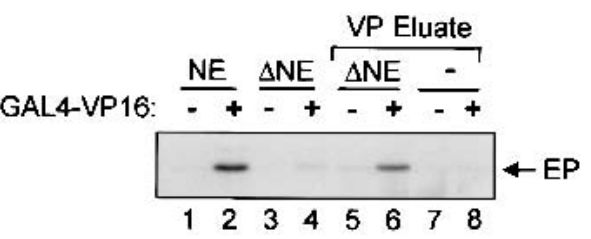

the purified hMed fraction. (B) Purified hMed complements $\Delta$ NE for activated transcription. Twenty nanograms of $\mathrm{pG}_{5} \mathrm{E}_{4} \mathrm{~T}$ template (lanes 1-6) was incubated with $35 \mu \mathrm{g} \Delta \mathrm{NE}$ (lanes 1-4) and (lanes 3,4) or 1 unit purified hMed (see Materials and Methods) (lanes 5,6) in the absence (lanes 1,3,5) or presence (lanes 2,4,6) of 5 ng GAL4-VP16. Transcription was measured by primer extension (arrow). Note that hMed alone cannot support transcription. GAL4-VP16-induced transcription is detected only when purified hMed is supplemented with $\Delta \mathrm{NE}$ (lane 4). (C) The VP eluate complements $\Delta$ NE. pG $_{5}$ E4T (lanes 1-8) was incubated with $35 \mu \mathrm{g}$ HeLa NE (lanes 1,2),

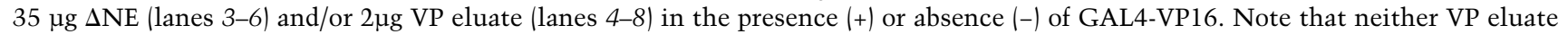
nor $\triangle \mathrm{NE}$ alone support transcription but the combination is active. 
that the association of RNA PolII with the purified hMed is substoichiometric (Malik and Roeder 2000). Purified hMed alone is unable to support either basal or activated transcription (Fig. 2B, lanes 5 and 6) on a plasmid template. We also used an alternative method to isolate the mediator coactivator complex: a GST-VP16 affinity purification approach described by Tjian and colleagues (Naar et al. 1999). This is a somewhat more complicated mediator fraction that, in our hands, contains p300, SWI/ SNF, and some TFIIB (data not shown). Mutations in the VP16 activation domain nearly eliminate coactivator association, demonstrating that purification of the complex is dependent on an intact activation domain (data not shown; Naar et al. 1999). This fraction will herein be designated "VP eluate." The VP eluate alone, like the purified hMed described above, cannot support basal or activated transcription on plasmid templates (Fig. 2C, lanes 7,8$)$.

To assess the activity of our purified hMed, we generated a mediator-depleted HeLa nuclear extract $(\Delta \mathrm{NE})$. $\Delta \mathrm{NE}$ is specifically depleted of mediator complex subunits; GTFs, including TFIID, are not depleted (Fig. 2A, cf. lane 1 [starting $\mathrm{NE}]$ and lane $2[\Delta \mathrm{NE}])$. $\Delta \mathrm{NE}$ alone is unable to support activated transcription (Fig. 2B, lanes $1,2$, Fig. $2 \mathrm{C}$, lanes 3,4$)$. Importantly, $\Delta \mathrm{NE}$ is unable to support activated transcription at all concentrations tested-up to $80 \mu \mathrm{g}$, the limit of our assay (data not shown).

Figure 2B demonstrates that our purified hMed is active. hMed can complement $\Delta \mathrm{NE}$, thereby enabling GAL4-VP16-activated transcription on $\mathrm{pG}_{5} \mathrm{E} 4 \mathrm{~T}$ templates (Fig. 2B, lanes 3,4). Figure 2C illustrates that the VP eluate contains an activity, which, in our assay, is functionally indistinguishable from hMed. Neither the VP eluate nor the $\triangle \mathrm{NE}$ fractions alone can support activation by GAL4-VP16 (Fig. 2C, lanes 4,8) but, when combined, they enable activated transcription (Fig. 2C, lanes $5,6)$. Having established that our purified hMed can support activation, we assessed whether activators directly recruit hMed to promoter templates.

\section{Purified human mediator is directly recruited by GAL4-VP16 to promoter DNA}

Figure $3 \mathrm{~A}$ demonstrates that purified hMed is directly recruited to immobilized $\mathrm{G}_{5} \mathrm{E} 4 \mathrm{~T}$ promoter DNA by GAL4-VP16. This recruitment assay was performed in parallel to an in vitro transcription assay using the same immobilized template. Purified hMed can support GAL4-VP16-induced transcription on $\mathrm{G}_{5} \mathrm{E} 4 \mathrm{~T}$ immobilized templates when incubated with mediator-depleted nuclear extract (Fig. 3B, lanes 3,4). Therefore, recruitment of hMed by GAL4-VP16 correlates with hMed-dependent, GAL4-VP16-induced transcription. As GAL4VP16 has been observed to have only a modest effect on the recruitment of TFIID to promoter DNA, we sought to determine whether recruitment of hMed by GAL4VP16 to a DNA template might affect TFIID recruitment. Alternately stated, is recruitment of TFIID and hMed cooperative?

\section{Recruitment of the human mediator from nuclear extract is affected by TFIID}

To begin our study of cooperative recruitment of hMed and TFIID in activated transcription, we used an immobilized template without a functional TATA box to examine the effect of reduced TFIID binding on hMed recruitment from HeLa nuclear extract. Our initial experiments revealed that under conditions where GAL4-VP16 is prebound and, thus, fully saturates the immobilized templates, hMed was equivalently recruited to both wild-type and TATA mut templates (data not shown). This result is consistent with that of others (Ranish et al. 1999) and is not surprising given the substantial interaction between VP16 and hMed (Fig. 3B; Naar et al. 1999). To observe an effect of TATA mut on hMed recruitment, we modified our assay conditions. Rather than prebinding GAL4-VP16, we incubated the activator in batch with the nuclear extract at a level optimal for in vitro transcription assays, which is, however, suboptimal
A

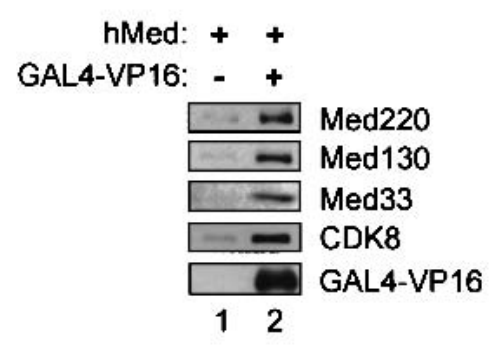

B
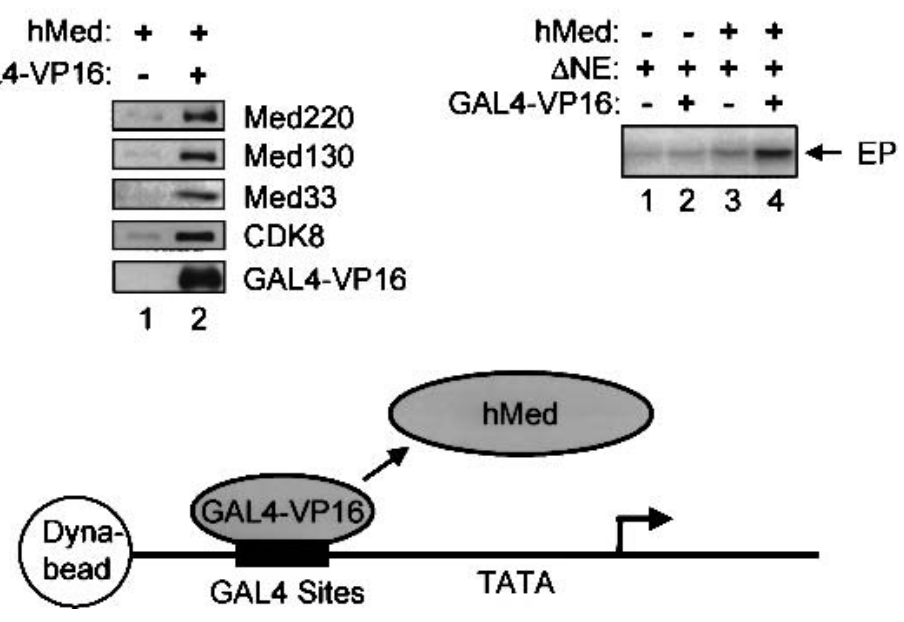

Figure 3. hMed is recruited by GAL4-VP16 on immobilized templates. (A) GAL4-VP16 stimulates recruitment of purified hMed to immobilized DNA templates. One unit purified hMed was incubated with $5 \mathrm{ng}$ purified, recombinant GAL4-VP16 and $10 \mathrm{fmol}$ immobilized $\mathrm{G}_{5} \mathrm{E} 4 \mathrm{~T}$ DNA. Bound protein is detected by immunoblot. (B) Purified hMed is required for activation on immobilized templates. Twenty nanograms of $\mathrm{pG}_{5} \mathrm{E} 4 \mathrm{~T}$ template (lanes 1-4) was incubated with $35 \mu \mathrm{g} \Delta \mathrm{NE}$ (lanes 1-4) and (lanes 3,4) 1 unit purified hMed in the absence (lanes 1,3) or presence (lanes 2,4) of 5 ng GAL4VP16. A phosphorimager scan of the gel is shown. 
with respect to GAL4-VP16 binding site saturation on these templates (data not shown). We assayed, by in vitro transcription, the activity of HeLa nuclear extract on these immobilized templates under these modified conditions. Figure 4A demonstrates a reduction in both basal and activated transcription on the TATA mut $_{\text {tem- }}$ plate relative to the wild-type template. An assay for recruitment of the transcription machinery was performed in parallel with this in vitro transcription assay. Predictably, we observed a significant reduction in TBP recruitment to the TATA mut $_{\text {muta }}$ templat 4B, cf. lanes 1 and 2). With the reduction in TBP binding on the TATA $_{\text {mut }}$ template, we observe a concomitant reduction in hMed recruitment (Fig. 4B, cf. lanes 1 and 2). Not unexpectedly, a decrease in TBP/TFIID recruitment to the TATA $_{\text {mut }}$ template also results in a decrease in GAL4-VP16 binding. Cooperative recruitment of TFIID and activators has been extensively studied (Sawadogo and Roeder 1985; Horikoshi et al. 1988a,b; Lieberman and Berk 1994; Chi et al. 1995; Ellwood et al. 1999). Consistent with these reports, lowering the concentration of TFIID decreases the affinity of GAL4-VP16 for

A

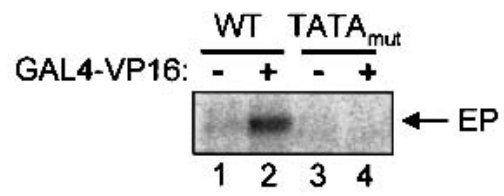

B

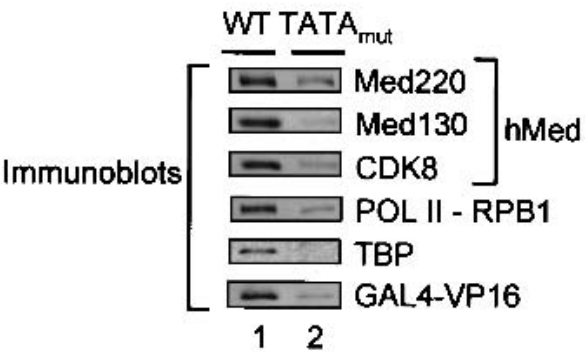

C

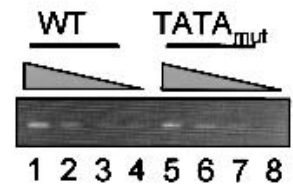

Figure 4. Cooperative assembly of transcription complexes requires a TATA box. $(A)$ In vitro transcription of $\mathrm{G}_{5} \mathrm{E} 4 \mathrm{~T}$ requires a TATA box. HeLa NE (lanes 1-4) was incubated with $40 \mathrm{fmol}$ WT $\mathrm{G}_{5}$ E4T immobilized template (lanes 1,2) or $40 \mathrm{fmol}$ TATA $_{\text {mut }} \mathrm{G}_{5}$ E4T immobilized template (lanes 3,4). No transcription signal is detectable from the TATA mut $_{\text {template. }}(B)$ Recruitment of hMed by GAL4-VP16 to $\mathrm{G}_{5} \mathrm{E} 4 \mathrm{~T}$ immobilized templates is dependent on a TATA box. HeLa nuclear extract was added with $5 \mathrm{ng}$ GAL4-VP16 (lanes 1,2) to $5 \mathrm{fmol}$ immobilized WT (lane 1) or TATA mut (lane 2) template. The presence of hMed subunits and GTFs was detected by immunoblot analysis. (C) Quantitation of immobilized templates. HindIII digests of WT or TATA mut $_{5}$ E4T immobilized templates were electrophoresed on a $1.5 \%$ agarose gel and stained with ethidium bromide. Four steps of twofold dilutions of the WT and TATA mut digests are shown adjacent to each other (WT, lanes 1-4; TATA $_{\text {mut }}$ lanes 5-8).
GAL4-DNA binding sites in footprinting assays using $\mathrm{G}_{5} \mathrm{E} 4 \mathrm{~T}$ templates (data not shown). The confounding effect of cooperativity between GAL4-VP16 and TFIID prevents us from determining whether the effect of reduced TFIID binding on the recruitment of hMed is direct, due to communication between TFIID and hMed, or if it is an indirect effect of the reduced recruitment of GAL4VP16. However, the results presented in Figure 4B are suggestive of a higher-order connection between the TFIID and hMed coactivator complexes.

\section{Cooperative recruitment of TFIID and human mediator to promoter DNA}

A higher-order assembly of coactivators and activators might form as a result of a series of cooperative proteinprotein and protein-DNA interactions (Johnson et al. 2001). A key aspect of this model is the potential for direct communication between hMed and TFIID coactivators. To determine if TFIID and hMed can stimulate each other's recruitment to promoter DNA, we modified our immobilized template assay protocol to remove any impact of cooperative effects on GAL4-VP16 binding. By prebinding high, saturating levels of GAL4-VP16 to the immobilized template, we were able to study, in isolation, the potential for cooperative recruitment of purified TFIID and human mediator.

The activity of the immunopurified TFIID used in our cooperative recruitment assays was assessed in an hMeddependent system. The in vitro transcription assay depicted in Figure 5A demonstrates that both immunopurified TFIID and purified hMed are required for restoration of GAL4-VP16-induced transcription in nuclear extract that is depleted of both TFIID and hMed (Fig. 5A, cf. lane 18 to lanes $12,14,16)$.

Figure 5B demonstrates that purified hMed can stimulate recruitment of purified TFIID to immobilized promoter DNA. TFIID was incubated with immobilized templates in the absence (Fig. 5B, lane 1) or presence (Fig. $5 \mathrm{~B}$, lane 2) of saturating levels of GAL4-VP16. Levels of TFIID recruitment in these first two lanes are below the detectable limit of the immunoblot. However, when the same amount of TFIID is incubated in the presence of saturating levels of purified hMed (Fig. 5B, lanes 5,6), TFIID recruitment in the presence of GAL4-VP16 is substantially increased (Fig. 5B, cf. lanes 2 and 6). The amount of TFIID used in this experiment is suboptimal with respect to TFIID template occupancy in the absence of hMed (data not shown). However, in the presence of hMed, TFIID and hMed are recruited to the immobilized template in roughly equimolar amounts (Fig. 5B). We also performed the experiment with an hMed fraction lacking PolII (a gift from T. Boyer and A. Berk) and observed a similar cooperative effect on TFIID binding (data not shown).

A model for cooperative interactions between TFIID and hMed implies the potential for mutual stabilization of these coactivator complexes on the DNA. Alternatively stated, if hMed can stimulate recruitment of TFIID to promoter DNA then the converse must also be 
A

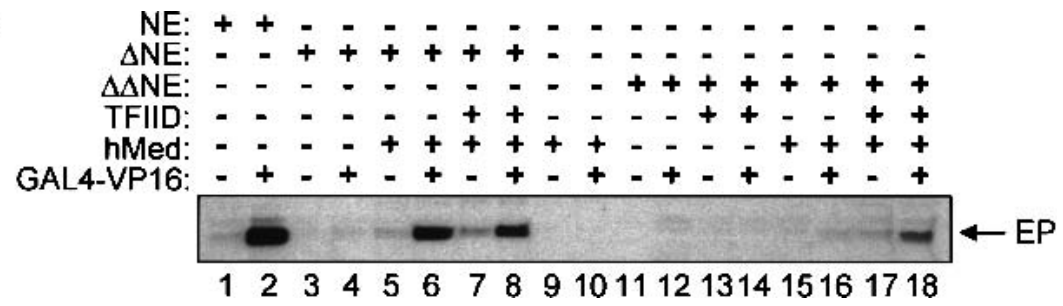

Figure 5. Human mediator and TFIID bind cooperatively to promoter DNA. (A) Purified hMed and TFIID are active in standard in vitro transcription reactions. Mediator-depleted, TFIID-depleted nuclear extract $(\Delta \Delta \mathrm{NE})$ was generated by heat-treatment of $\Delta \mathrm{NE}$. The transcription reactions contained $20 \mathrm{ng} \mathrm{pG}_{5} \mathrm{E} 4 \mathrm{~T}$ with $(+)$ or without (-) 5 ng GAL4-VP16 and $48 \mu \mathrm{g} \mathrm{HeLa}$

B

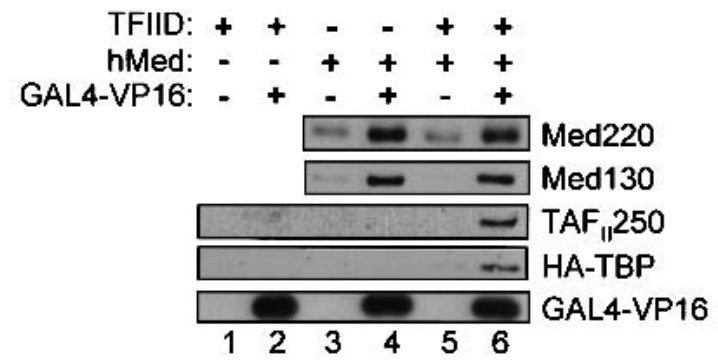

\begin{tabular}{|c|c|c|c|c|}
\hline Protein & $\begin{array}{c}\mathrm{ng} \\
\text { Bound to } \\
\text { Template }\end{array}$ & $\begin{array}{c}\text { MW } \\
(\mathrm{kDa})\end{array}$ & $\begin{array}{c}\text { fmole } \\
\text { Bound to } \\
\text { Template }\end{array}$ & $\begin{array}{c}\text { Stoichiometry } \\
\text { Prot / } \\
\text { Template }\end{array}$ \\
\hline GAL4-VP16 & 5 & 25 & 200 & 8 \\
\hline Med220 & 4 & 220 & 18 & 0.7 \\
\hline Med130 & 3 & 130 & 20 & 0.8 \\
\hline TBP & 0.6 & 38 & 16 & 0.6 \\
\hline
\end{tabular}

C

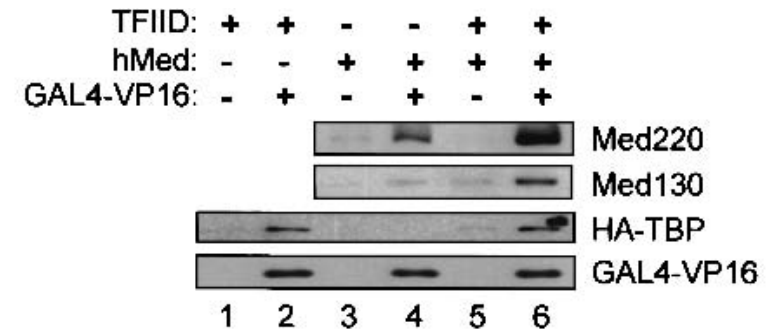

D

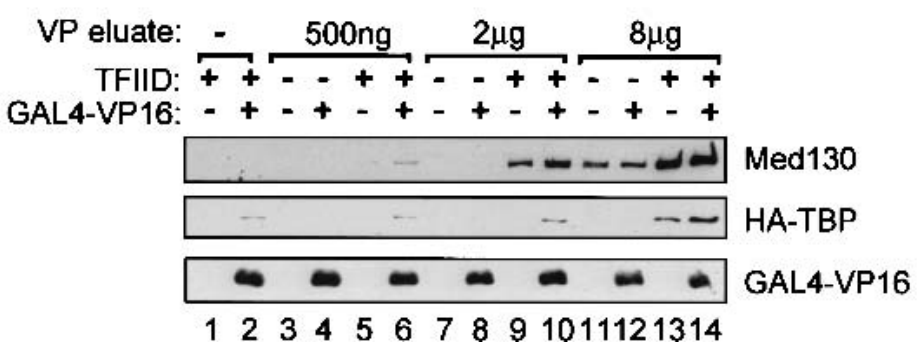
NE (lanes 1,2), $36 \mu \mathrm{g} \Delta \mathrm{NE}$ (lanes 3-8), or $35 \mu \mathrm{g}$ $\Delta \Delta \mathrm{NE}$ (lanes 11-18) in the presence of $120 \mathrm{ng}$ TFIID (lanes $7,8,13,14,17,18)$ and 1 unit hMed (lanes 5-10,15-18). Transcription was analyzed by primer extension (arrow). An autoradiograph of the gel is shown. $(B)$ Saturating levels of purified hMed recruit TFIID to immobilized templates. Twenty-five fmol $\mathrm{G}_{5} \mathrm{E} 4 \mathrm{~T}$ immobilized template (every lane) was prebound with $200 \mathrm{ng}$ GAL4-VP16 (+) in every other lane. Subsequently, $40 \mathrm{ng}$ TFIID and $12 \mathrm{ng}$ TFIIA were added to lanes 1, 2, 5, and 6. In lanes 3-6, 0.8 units hMed were added. After washing, bound proteins were detected by immunoblot analysis. The approximate stoichiometry of GAL4-VP16, TFIID, and hMed recruitment is 10 (5 GAL4VP16 dimers):1:1, respectively (see Materials and Methods). (C) Saturating levels of TFIID stimulate hMed recruitment to immobilized templates. Twenty-five fmol $\mathrm{G}_{5} \mathrm{E} 4 \mathrm{~T}$ templates were prebound with 200 ng GAL4-VP16 in every other lane. One hundred-twenty nanograms of TFIID and 12 ng TFIIA were added to lanes 1 , 2, 5, and 6; 0.04 units hMed were added to lanes 3-6. Protein bound to the immobilized templates was detected by immunoblot. $(D)$ hMed (from the VP eluate) and purified TFIID bind cooperatively to immobilized $\mathrm{G}_{5} \mathrm{E} 4 \mathrm{~T}$ templates. Immobilized template recruitment assay: 25 fmol of immobilized $\mathrm{G}_{5} \mathrm{E} 4 \mathrm{~T}$ was preincubated for $20 \mathrm{~min}$ at room temperature in the presence $(+)$ and absence (-) of 200 ng GAL4-VP16 with indicated amounts of VP eluate, and/or TFIID (40 ng) and TFIIA (12 ng). After $30 \mathrm{~min}$, unbound protein was removed by washing and the bound protein was resolved on $4 \%-15 \%$ SDS-PAGE. Immunoblots of bound protein are shown. Med130 is used to measure hMed binding; TBP (HA) is representative of TFIID binding. Immunoblots with other subunits of these protein complexes demonstrate identical results. true: TFIID must be able to stimulate hMed recruitment. Demonstration of this proof of principle in experiments with our highly purified hMed required a modification of the recruitment assay conditions. Specifically, we used a higher concentration of TFIID and a lower concentration of hMed. Figure $5 \mathrm{C}$ depicts an experiment where, in the presence of GAL4-VP16, TFIID is saturating with respect to both template occupancy and transcriptional activity. Comparison of lanes 4 and 6 of Figure 5C demonstrates that saturating levels of TFIID stimulate the recruitment of subsaturating levels of hMed. The concentration of hMed used in this experiment is substantially below the optimal concentration used in the experiments shown in Figure $5 \mathrm{~B}$ and $2 \mathrm{~B}$. It is our observation that GAL4-VP16 has a strong stimulatory effect on the recruitment of hMed and a relatively weak impact on the recruitment of TFIID. As such, we needed to manipulate the principle of mass-action and use substantially low concentrations of hMed in the experiment shown in Figure 5C to demonstrate that TFIID is capable of stimulating recruitment of hMed to an activator-bound template.

Our alternatively purified hMed fraction, the VP eluate, was also assayed for its ability to stimulate recruitment of TFIID to DNA. We demonstrate in Figure 5D 
that an increase in the concentration of VP eluate added to the immobilized template reaction results in a concomitant increase in TFIID recruitment (Fig. 5D, cf. lane 2 and lanes 10,14). Conversely, incubation of TFIID with the VP eluate stimulates hMed recruitment to the immobilized template (Fig. 5D, cf. lanes 4 and 6, lanes 8 and 10 , and lanes 12 and 14).

Figure 5D also demonstrates that at high levels of VP eluate, hMed binds to DNA independently of activator. This "basal" hMed binding is stimulated by TFIID (Fig. 5D, cf. lanes 7 and 9, and lanes 11 and 13) and, conversely, it stimulates TFIID binding (Fig. 5D, cf. lanes 1 and 13). These effects in the absence of activator are further demonstration of cooperative interactions between the TFIID and hMed coactivators.

\section{Preassembly of a complex containing both TFIID and hMed stimulates transcription}

There are several recent reports about the effects of the mediator complex on basal transcription (Mittler et al. 2001; Baek et al. 2002). An examination of the functional relevance of our TFIID/hMed basal complex is depicted in Figure 6A. TFIID alone, hMed alone, or both were preincubated for $30 \mathrm{~min}$ followed by a 4-min transcription reaction in the mediator-depleted extract. Preincubation of TFIID and hMed together results in a stimulation of transcription (Fig. 6A, cf. lane 3 to lanes 1,2,4). This indicates that formation of a TFIID, hMed complex is a rate-limiting step in basal transcription and establishes functional relevance of a TFIID/hMed coactivator complex.

Figure 6B demonstrates that preassembly of the TFIID/ hMed complex also stimulates activated transcription. A TFIID-depleted, mediator-depleted nuclear extract $(\Delta \Delta \mathrm{NE})$ was generated by immunodepletion of TBP, $\mathrm{TAF}_{\mathrm{II}} 250$, and $\mathrm{TAF}_{\mathrm{II}} 32$ from mediator-depleted $(\Delta \mathrm{NE})$ HeLa nuclear extract. $\Delta \Delta$ NE is dependent on both TFIID and hMed for activated transcription (Fig. 6B, lanes 1,2). After a 15-min preincubation of GAL4-VP16 and $\Delta \Delta \mathrm{NE}$ with TFIID alone (Fig. 6B, lane 3), hMed alone (Fig. 6B, lane 4), or both together (Fig. 6B, lane 5), first-round transcription was assessed. Preassembly of both TFIID and hMed in the presence of GAL4-VP16 stimulates transcription (Fig. 6B, cf. lane 5 to lanes $3,4,6$ ). This provides evidence that formation of an activator/TFIID/hMed higher-order complex enhances the efficiency of PIC assembly, and thus plays an important role in activated transcription.

\section{Discussion}

Studies using GAL4-VP16 have provided significant insight into biochemical mechanisms of gene activation (Carey et al. 1990b). Like many activators, GAL4-VP16 accurately stimulates high levels of transcription from model templates, both in vitro (Carey et al. 1990a) and in vivo (Sadowski et al. 1988), on both naked (Carey et al. 1990b) and chromatin templates (Pazin et al. 1994). Early studies demonstrated that GAL4-VP16 stimulated transcription via assembly of active transcription complexes

A
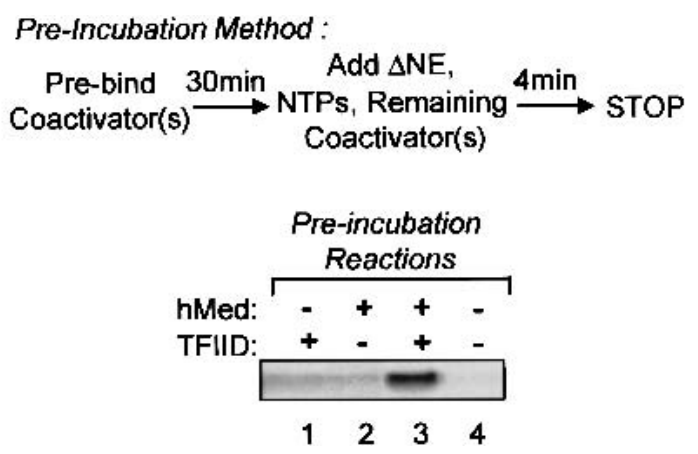

B

Pre-incubation Method :

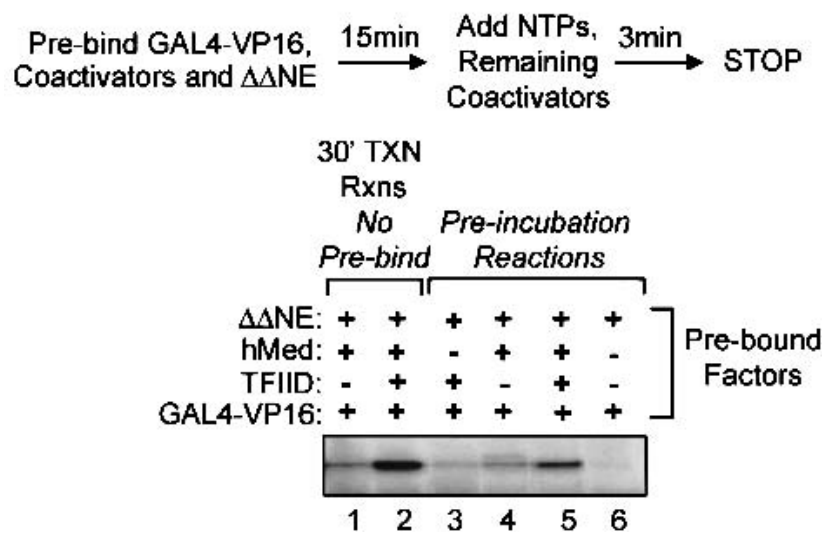

Figure 6. Preassembly of a complex containing both TFIID and hMed stimulates transcription. (A) Preincubation of TFIID and hMed in the absence of activator enhances basal transcription. The experimental design is outlined at top. The $60-\mu \mathrm{L}$ reaction mixtures contained $20 \mathrm{ng} \mathrm{pG}_{5} \mathrm{E} 4 \mathrm{~T}$ and, as indicated, $160 \mathrm{ng}$ TFIID, 12 ng TFIIA, or 2 units hMed. Where indicated in the figure, these factors were preincubated (TFIIA was added along with TFIID). Salt and buffer concentrations were held constant throughout the reaction. Thirty-five micrograms of $\Delta \mathrm{NE}$, NTPs, and coactivators were added to the preincubation mixes for 4 min at $30^{\circ} \mathrm{C}$ and transcription was measured by primer extension analysis. An autoradiograph of the gel is shown. (B) Preincubation of TFIID and hMed in the presence of GAL4-VP16

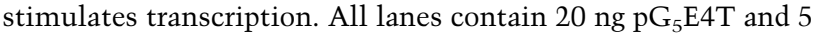
ng GAL4-VP16. Lanes 1 and 2 are standard, 30-min in vitro transcription reactions. Addition of 2 units hMed in lane 2 restores activated transcription. Lanes 1 and 2 contain $35 \mu \mathrm{g}$ mediator-depleted, TFIID-depleted nuclear extract $(\Delta \Delta \mathrm{NE})$ and 2 units hMed. The addition of 160 ng TFIID and 12 ng TFIIA in lane 2 restores activated transcription. $\triangle \Delta \mathrm{NE}$ was generated by immunodepletion of $\mathrm{TBP}, \mathrm{TAF}_{\mathrm{II}} 250$, and $\mathrm{TAF}_{\mathrm{II}} 32$ and is greater than 80 -fold depleted of these subunits. The experimental design of preincubation reactions (lanes 3-6) is outlined at top. Preincubation, in vitro transcription conditions were the same as those described in panel $A$ with the following exceptions: (1) preincubation was performed in the presence of GAL4-VP16 and $35 \mu \mathrm{g} \Delta \Delta \mathrm{NE}_{\text {; }}(2)$ preincubation was for $15 \mathrm{~min}$; (3) the second incubation with remaining factors and NTPs was for $3 \mathrm{~min}$. Shown is a cropped image of an autoradiograph; all lanes are from the same gel. 
(Wang et al. 1992a). Recruitment of TFIID and activatorstimulated conformational changes were suggested to be rate-limiting in activated transcription in vitro (Horikoshi et al. 1988b; Wang et al. 1992b; Chi et al. 1995; Chi and Carey 1996). GAL4-VP16 has a direct effect on TFIID recruitment, but this effect has been shown to be modest relative to other activators (Chi et al. 1995). If stable recruitment of TFIID to the promoter is a critical aspect of activator mechanism, how then does GAL4-VP16 fulfill this role? We propose that GAL4-VP16 directly recruits the human mediator complex, and that cooperative interactions between TFIID and hMed indirectly result in efficient GAL4-VP16 recruitment of TFIID.

GAL4-VP16 interacts with both TFIID and hMed directly. In the case of TFIID, the proposed subunit targets include both TBP and $\mathrm{TAF}_{\mathrm{II}} 32$ (Klemm et al. 1995). The proposed hMed target of GAL4-VP16 is Med78/TRAP80 (Ito et al. 1999). Our data support the functionality of this proposed GAL4-VP16-hMed interaction, as we have demonstrated that GAL4-VP16 directly recruits the human mediator coactivator complex to promoter DNA under conditions where purified mediator can support transcription. The novel contribution of this study is the demonstration of direct communication between the hMed and TFIID coactivators, which can lead to their cooperative recruitment to promoter DNA. We provide evidence that the direct recruitment of hMed by GAL4VP16 can enhance recruitment of TFIID. As would be expected of truly cooperative interactions, we were able to demonstrate that the opposite effect is also possible; that is, that TFIID can stimulate hMed recruitment.

An interesting aspect of our study was the discovery of basal recruitment of the TFIID and hMed coactivator complexes. We believe this result supports our assertion that TFIID and hMed directly communicate. If, as we argue, the combination of TFIID and hMed recruitment to DNA serves as a scaffold for assembly of the PIC, we would expect to see that preassembly of TFIID and hMed in the absence or presence of activator would stimulate the rate of transcription (Johnson et al. 2001). Indeed, assembly of a higher-order complex, which includes TFIID and hMed in the presence or absence of GAL4VP16, stimulates transcription. This lends credence to the idea that cooperative assembly is of functional relevance.

Several previous studies have indicated the possibility of an interaction between TFIID and hMed. Two recent studies from the Roeder group report the existence of transcriptional synergy between mediator and TAFs (Guermah et al. 2001; Baek et al. 2002). They propose a "close mechanistic linkage between [TAFs and mediator] that most likely operates at the level of combined effects on the general transcription machinery" (Baek et al. 2002). Several older studies on the effects of activators on TFIID, when viewed in light of current knowledge of the critical nature of the mediator, also imply the potential for direct TFIID-mediator interactions.

The mammalian activator, SP1, contains a glutaminerich activation domain that interacts directly with TFIID in a manner critical for in vitro activation (Hoey et al.
1993; Chen et al. 1994). CRSP, a highly active mediator fraction, was isolated as an activity required for SP1 in vitro activation (Ryu et al. 1999). In another study demonstrating an alternative method of mediator purification, it was shown that hMed does not directly interact with SP1 (Naar et al. 1999). SP1 directly recruits only TFIID, but both TFIID and the hMed are required for SP1 activation (Ryu et al. 1999). How, then, is the mediator complex brought to the promoter? Viewed in light of our model, we propose that TFIID can interact with the mediator complex, and thus recruit it to the promoter.

Our own studies with the Epstein-Barr virus activator, ZEBRA, show that it cooperatively recruits purified TFIID to promoter DNA (Chi et al. 1995) but does not strongly interact with purified hMed (data not shown), which is required for activation in in vitro transcription assays. As ZEBRA does not directly recruit hMed, we propose that the cooperative recruitment of TFIID by ZEBRA, through interactions with multiple TAFs, TBP, or TFIIA, is responsible for recruitment of hMed. Buttressing this idea, older studies on the mechanism of ZEBRA and the IFN- $\beta$ enhanceosome indicate that the USA fraction, which contains the mediator, is required along with DAB complex for stable PIC assembly (Lieberman 1994; Kim and Maniatis 1997). Formation of this stable, higher-order coactivator complex, similar to what was seen as a reinitiation scaffold by Hahn and colleagues (Yudkovsky et al. 2000), may be the true ratelimiting step in activation.

A further indication of cooperative assembly of the yeast mediator and TFIID comes from CHIP assays by the Struhl (Kuras and Struhl 1999) and Green groups (Li et al. 1999, 2000) and immobilized template recruitment experiments by Hahn and colleagues (Ranish et al. 1999). Studies designed to evaluate the requirements of TAFs at individual promoters in vivo demonstrate that TBP binding at a promoter can be dependent on functional yeast mediator subunits (Kuras and Struhl 1999; Li et al. 1999, 2000). Additionally, Ranish et al. (1999) indirectly demonstrated that the model activator GAL4-AH does not strongly interact with the yeast mediator but nevertheless recruits it to an immobilized template in a TFIID (and TFIIA)- dependent manner. Further, Ranish et al. (1999) indicate that some GTFs, including TFIIB, are required to facilitate this recruitment, suggesting that these GTFs may help stabilize the assembly when the mediator is recruited through TFIID. This may be reflective of the requirement for TFIIB in the ZEBRA and IFN$\beta$-induced formation of the stable USA/TFIID/TFIIA coactivator complex (Lieberman 1994; Kim and Maniatis 1997). We demonstrate in our present study, however, that TFIIB is not absolutely required for the direct interaction between TFIID and hMed, as our purified hMed does not contain TFIIB.

We propose a model for transcriptional activation in which a series of cooperative, compensatory interactions between activators, coactivators, and promoter DNA occur (Lehman et al. 1998), leading to formation of a higher-order assembly, which is composed minimally of activator(s), TFIID, and the human mediator complex 
Figure 7. Model of cooperative recruitment of coactivators to DNA templates. Our hypothesis is that reciprocal, cooperative interactions between activator (or series of activators), TFIID, hMed, and template DNA determine the stability of a higher-order nucleoprotein complex. The presence of some form of this higher-order assembly is required for all activated transcription. A key feature of this model is direct communication between the hMed and TFIID.

(Fig. 7). This cooperative assembly model allows for flexibility in how regulatory sequences surrounding the promoter may enable assembly of this higher-order coactivator complex; both DNA-protein interactions and protein-protein interactions are variables that may impact cooperative assembly (Johnson et al. 2001). The subunit compositions of TFIID and hMed and the sequences within the core promoter could modulate the order in which the complex assembles. The chromatin status of a gene and its regulated remodeling program (Cosma et al. 1999; Krebs et al. 1999; for review, see Fry and Peterson 2001) may also influence accessibility of TFIID (Lomvardas and Thanos 2001) or activators to DNA, thus further influencing the way in which the PIC assembles. Indeed, a combination of all these influences enables tremendous diversity in the way activators could function in vivo (Johnson et al. 2001).

Synergistic activation of a gene in vivo may involve concerted chromatin remodeling and PIC assembly. However, synergy is observed in vitro on naked DNA templates (Carey et al. 1990b). One can imagine various scenarios for how such synergistic gene activation could be achieved within the framework of this model. Multiple, simultaneous contacts of activators with the different subunits of mediator (Ito et al. 1999) may cooperatively recruit hMed, and, in so doing, facilitate TFIID binding. Conversely, multiple activator contacts with different TAFs, TBP, or TFIIA could recruit TFIID /Chi et al. 1995; Sauer et al. 1995), which then recruits hMed. Finally, one activator could interact with TFIID and another with mediator, as has been proposed for the synergy of activation by SREBP and SP1 on the LDLR promoter (Naar et al. 1998). The collection of studies reviewed herein, when viewed in light of the data presented in the present study, indicate that cooperative recruitment of TFIID and hMed may be a critical aspect of the mechanism of action of many activators.

\section{Materials and methods}

\section{Factor purification}

Purification of recombinant GAL4-VP16 (Tantin et al. 1996), recombinant TFIIA (Ozer et al. 1994), and hemagglutinin (HA) epitope-tagged TFIID from the HeLa cell line LTR $\alpha 3$ (Zhou et al. 1992) was as described previously. HeLa nuclear extracts were prepared as described (Dignam et al. 1983). Human mediator was prepared essentially as described (Rachez et al. 1998) except that it was purified from HeLa cell nuclear extract and was eluted with a Med220 (DRIP205) NR2C peptide (Rachez et al. 2000). One unit of hMed is defined as the amount required to complement $35 \mu \mathrm{g}$ of $\Delta \mathrm{NE}$ to produce the same fmol amount of transcript as that produced by $35 \mu \mathrm{g}$ HeLa NE. VP16 eluate was purified from HeLa nuclear extract via a GST-VP16 affinity resin as described except that bound proteins were eluted without Sarkosyl (Naar et al. 1999).

Mediator-depleted HeLa nuclear extract $(\Delta \mathrm{NE})$ was prepared by affinity depletion chromatography. Three milliliters of Glutathione-Sepharose Fast-Flow 4B (Amersham) beads, bound by GST-TR $_{\text {LBD }}$ at $0.3 \mathrm{mg}$ protein $/ \mathrm{mL}$ of beads, were packed onto a $5-\mathrm{mL}$ column and charged for $1 \mathrm{~h}$ with $10 \mu \mathrm{M} \mathrm{T}$ ligand in $0.1 \mathrm{M}$ $\mathrm{KCl}$ binding buffer (Rachez et al. 1998). Twenty-four milligrams of HeLa nuclear extract $\left(+10 \mu \mathrm{M} \mathrm{T}_{3}\right)$ in $3 \mathrm{~mL}$ was loaded onto the column at a flow rate of $3 \mathrm{~mL} / \mathrm{h}$. Peak fractions were identified by Bradford assays, pooled, and analyzed by both immunoblot and in vitro transcription. The extent of hMed depletion was determined by serial dilution and immunoblot.

Mediator-depleted, TFIID-depleted HeLa nuclear extract $(\Delta \Delta \mathrm{NE})$ was prepared by immunoaffinity depletion of the mediator-depleted HeLa nuclear extract $(\Delta \mathrm{NE})$. Forty $\mu \mathrm{L}$ protein $\mathrm{G}$ Sepharose beads (Amersham) were incubated with a cocktail of $20 \mu \mathrm{TAF}_{\text {II }} 250$ (Santa Cruz), $20 \mu \mathrm{g} \mathrm{TAF}_{\mathrm{II}} 32$ (Santa Cruz), and 20 $\mu \mathrm{g}$ TBP (Santa Cruz) antibodies for $1 \mathrm{~h}$ at room temperature. The beads were washed 3 times in buffer D (Dignam et al. 1983) and incubated with $800 \mu \mathrm{g} \Delta \mathrm{NE}$ for $2 \mathrm{~h}$ at $4^{\circ} \mathrm{C}$. The supernatant was collected and assayed by immunoblot and in vitro transcription. The extent of depletion of TBP, $\mathrm{TAF}_{\mathrm{II}} 250$, and $\mathrm{TAF}_{\mathrm{II}} 32$ was determined by serial dilution and immunoblot. This approach is similar to that used by Oelgeschlager et al. (1998).

\section{Immobilized template recruitment and in vitro transcription assays}

Immobilized templates were generated essentially as described (Kim et al. 1998; Ranish et al. 1999; Yie et al. 1999). Biotinylated fragments were amplified from $\mathrm{pG}_{5} \mathrm{E} 4 \mathrm{~T}$ (Carey et al. 1990a) with a 27 -nucleotide, biotinylated primer positioned $205 \mathrm{bp}$ upstream of the GAL4 sites and with a downstream T7 sequencing primer. The 650-bp biotinylated PCR products were fractionated on agarose gels and purified using a QIAquick gel extraction kit (QIAGEN). The purified DNAs were bound to Dynabeads $^{\mathrm{TM}}$ (Dynal) in excess according to the manufacturer's instructions. Typically, we recovered 2 -fmol DNA per $\mu \mathrm{g}$ of beads. TATA $_{\text {mut }}$ immobilized DNA templates were amplified from plasmid templates generated by two-step PCR mutagenesis of $\mathrm{pG}_{5} \mathrm{E} 4 \mathrm{~T}$. Conversion of the six central alternating AT base pairs 
within the E4 TATA to a run of six CG base pairs was confirmed by DNA sequencing. Biotinylated TATA $_{\text {mut }}$ fragments were generated from $\mathrm{pG}_{5}$ E4T-TATA mut $_{\text {and bound to Dynabeads }}{ }^{\mathrm{TM}}$ as described above.

Immobilized template recruitment assays were performed as described (Ranish et al. 1999; Yie et al. 1999). Typically, the immobilized templates were incubated with factors indicated in the figure legends at room temperature in standard transcription buffer (Tantin et al. 1996) lacking nucleoside triphosphates (NTPs). The beads were immobilized on a magnetic particle concentrator (MPC), washed, and resuspended in SDS-PAGE loading dye. Factors bound to the template were detected by immunoblot. Details of template and protein concentrations are described in the figure legends. Antibodies to the following polypeptides were obtained from the following sources: PolII CTD 8WG16 (QED Bioscience), Med130/SUR2 (Pharmingen), TFIIB (Chi et al. 1995). All other antibodies were purchased from Santa Cruz Biotechnology.

The approximate stoichiometry of GAL4-VP16, hMed, and TFIID binding to immobilized templates was determined by the following: (1) Measurement of Med130, Med220, and TBP concentrations within their respective complexes by silver stain SDS-PAGE gel and comparison to BSA standards; (2) Determination of moles of protein bound to immobilized templates by immunoblot comparison to standard, known amounts of protein.

In vitro transcription and primer extension reactions with pG5E4T templates were performed as described (Tantin et al. 1996). Details of protein concentration and preincubation of factors are described in the figure legends. Heat-treated nuclear extracts were prepared by incubating $15 \mu \mathrm{L}$ aliquots of nuclear extracts at $43^{\circ} \mathrm{C}$ for $11 \mathrm{~min}$ as described (Nakajima et al. 1988). Transcription reactions using immobilized templates were performed using the standard transcription conditions as described for recruitment assays but with the addition of $0.5 \mathrm{mM}$ of each NTP. Transcripts were analyzed by primer extension as described (Tantin et al. 1996) and fractionated by electrophoresis on $10 \%$ polyacrylamide/urea gels electrophoresed in $1 \times$ TBE buffer. Autoradiographs or phosphorimager scans of the gels are shown.

\section{Acknowledgments}

We thank Erin Gutierrez, Norma Ibarra, and Wendy Lam for technical help, Jamie Tran for help with figure preparation, and Katherine Mitsouras, Anjali Jain, and JoAnn Zhang for critical discussion. We thank Arnie Berk and Tom Boyer for discussions and providing some reagents. This study was supported by grant GM057283 from the NIH. K.J. is supported by the USPHS National Research Service Award GM07185.

The publication costs of this article were defrayed in part by payment of page charges. This article must therefore be hereby marked "advertisement" in accordance with 18 USC section 1734 solely to indicate this fact.

\section{References}

Baek, H.J., Malik, S., Qin, J., and Roeder, R.G. 2002. Requirement of TRAP/mediator for both activator-independent and activator-dependent transcription in conjunction with TFIID-associated TAF(II)s. Mol. Cell. Biol. 22: 2842-2852.

Boyer, T.G., Martin, M.E., Lees, E., Ricciardi, R.P., and Berk, A.J. 1999. Mammalian Srb/mediator complex is targeted by adenovirus E1A protein. Nature 399: 276-279.
Buratowski, S., Hahn, S., Guarente, L., and Sharp, P.A. 1989. Five intermediate complexes in transcription initiation by RNA polymerase II. Cell 56: 549-561.

Carey, M., Leatherwood, J., and Ptashne, M. 1990a. A potent GAL4 derivative activates transcription at a distance in vitro. Science 247: 710-712.

Carey, M., Lin, Y.S., Green, M.R., and Ptashne, M. 1990b. A mechanism for synergistic activation of a mammalian gene by GAL4 derivatives. Nature 345: 361-364.

Chen, J.L., Attardi, L.D., Verrijzer, C.P., Yokomori, K., and Tjian, R. 1994. Assembly of recombinant TFIID reveals differential coactivator requirements for distinct transcriptional activators. Cell 79: 93-105.

Chi, T. and Carey, M. 1996. Assembly of the isomerized TFIIATFIID-TATA ternary complex is necessary and sufficient for gene activation. Genes \& Dev. 10: 2540-2550.

Chi, T., Lieberman, P., Ellwood, K., and Carey, M. 1995. A general mechanism for transcriptional synergy by eukaryotic activators. Nature 377: 254-257.

Cosma, M.P., Tanaka, T., and Nasmyth, K. 1999. Ordered recruitment of transcription and chromatin remodeling factors to a cell cycle- and developmentally regulated promoter. Cell 97: 299-311.

Dignam, J.D., Lebovitz, R.M., and Roeder, R.G. 1983. Accurate transcription initiation by RNA polymerase II in a soluble extract from isolated mammalian nuclei. Nucleic Acids Res. 11: 1475-1489.

Ellwood, K., Huang, W., Johnson, R., and Carey, M. 1999. Multiple layers of cooperativity regulate enhanceosome-responsive RNA polymerase II transcription complex assembly. Mol. Cell. Biol. 19: 2613-2623.

Flanagan, P.M., Kelleher III, R.J., Sayre, M.H., Tschochner, H., and Kornberg, R.D. 1991. A mediator required for activation of RNA polymerase II transcription in vitro. Nature 350: 436-438.

Fondell, J.D., Guermah, M., Malik, S., and Roeder, R.G. 1999. Thyroid hormone receptor-associated proteins and general positive cofactors mediate thyroid hormone receptor function in the absence of the TATA box-binding protein-associated factors of TFIID. Proc. Natl. Acad. Sci. 96: 1959-1964.

Fry, C.J. and Peterson, C.L. 2001. Chromatin remodeling enzymes: Who's on first? Curr. Biol. 11: R185-R197.

Gu, W., Malik, S., Ito, M., Yuan, C.X., Fondell, J.D., Zhang, X., Martinez, E., Qin, J., and Roeder, R.G. 1999. A novel human SRB/MED-containing cofactor complex, SMCC, involved in transcription regulation. Mol. Cell 3: 97-108.

Guermah, M., Tao, Y., and Roeder, R.G. 2001. Positive and negative TAF(II) functions that suggest a dynamic TFIID structure and elicit synergy with traps in activator-induced transcription. Mol. Cell. Biol. 21: 6882-6894.

Hahn, S. 1998. The role of TAFs in RNA polymerase II transcription. Cell 95: 579-582.

Hisatake, K., Hasegawa, S., Takada, R., Nakatani, Y., Horikoshi, M., and Roeder, R.G. 1993. The p250 subunit of native TATA box-binding factor TFIID is the cell- cycle regulatory protein CCG1. Nature 362: 179-181.

Hoey, T., Weinzierl, R.O., Gill, G., Chen, J.L., Dynlacht, B.D., and Tjian, R. 1993. Molecular cloning and functional analysis of Drosophila TAF110 reveal properties expected of coactivators. Cell 72: 247-260.

Holstege, F.C., Jennings, E.G., Wyrick, J.J., Lee, T.I., Hengartner, C.J., Green, M.R., Golub, T.R., Lander, E.S., and Young, R.A. 1998. Dissecting the regulatory circuitry of a eukaryotic genome. Cell 95: 717-728.

Horikoshi, M., Carey, M.F., Kakidani, H., and Roeder, R.G. 1988a. Mechanism of action of a yeast activator: Direct ef- 
fect of GAL4 derivatives on mammalian TFIID-promoter interactions. Cell 54: 665-669.

Horikoshi, M., Hai, T., Lin, Y.S., Green, M.R., and Roeder, R.G. 1988b. Transcription factor ATF interacts with the TATA factor to facilitate establishment of a preinitiation complex. Cell 54: 1033-1042.

Ito, M., Yuan, C.X., Malik, S., Gu, W., Fondell, J.D., Yamamura, S., Fu, Z.Y., Zhang, X., Qin, J., and Roeder, R.G. 1999. Identity between TRAP and SMCC complexes indicates novel pathways for the function of nuclear receptors and diverse mammalian activators. Mol. Cell 3: 361-370.

Johnson, K.M., Mitsouras, K., and Carey, M. 2001. Eukaryotic transcription: The core of eukaryotic gene activation. Curr. Biol. 11: R510-R513.

Kelleher, R.J., 3rd, Flanagan, P.M., and Kornberg, R.D. 1990. A novel mediator between activator proteins and the RNA polymerase II transcription apparatus. Cell 61: 1209-1215.

Kim, T.K. and Maniatis, T. 1997. The mechanism of transcriptional synergy of an in vitro assembled interferon-beta enhanceosome. Mol. Cell 1: 119-129.

Kim, T.K., Kim, T.H., and Maniatis, T. 1998. Efficient recruitment of TFIIB and CBP-RNA polymerase II holoenzyme by an interferon-beta enhanceosome in vitro. Proc. Natl. Acad. Sci. 95: 12191-12196.

Kim, Y.J., Bjorklund, S., Li, Y., Sayre, M.H., and Kornberg, R.D. 1994. A multiprotein mediator of transcriptional activation and its interaction with the C-terminal repeat domain of RNA polymerase II. Cell 77: 599-608.

Klemm, R.D., Goodrich, J.A., Zhou, S., and Tjian, R. 1995. Molecular cloning and expression of the $32-\mathrm{kDa}$ subunit of human TFIID reveals interactions with VP16 and TFIIB that mediate transcriptional activation. Proc. Natl. Acad. Sci. 92: 5788-5792

Krebs, J.E., Kuo, M.H., Allis, C.D., and Peterson, C.L. 1999. Cell cycle-regulated histone acetylation required for expression of the yeast HO gene. Genes \& Dev. 13: 1412-1421.

Kretzschmar, M., Stelzer, G., Roeder, R.G., and Meisterernst, M. 1994. RNA polymerase II cofactor PC2 facilitates activation of transcription by GAL4-AH in vitro. Mol. Cell. Biol. 14: 3927-3937.

Kuras, L. and Struhl, K. 1999. Binding of TBP to promoters in vivo is stimulated by activators and requires Pol II holoenzyme. Nature 399: 609-613.

Lehman, A.M., Ellwood, K.B., Middleton, B.E., and Carey, M. 1998. Compensatory energetic relationships between upstream activators and the RNA polymerase II general transcription machinery. J. Biol. Chem. 273: 932-939.

Li, X.Y., Virbasius, A., Zhu, X., and Green, M.R. 1999. Enhancement of TBP binding by activators and general transcription factors. Nature 399: 605-609.

Li, X.Y., Bhaumik, S.R., and Green, M.R. 2000. Distinct classes of yeast promoters revealed by differential TAF recruitment. Science 288: 1242-1244.

Lieberman, P. 1994. Identification of functional targets of the Zta transcriptional activator by formation of stable preinitiation complex intermediates. Mol. Cell. Biol. 14: 8365-8375.

Lieberman, P.M. and Berk, A.J. 1994. A mechanism for TAFs in transcriptional activation: Activation domain enhancement of TFIID-TFIIA-promoter DNA complex formation. Genes \& Dev. 8: 995-1006.

Lomvardas, S. and Thanos, D. 2001. Nucleosome sliding via TBP DNA binding in vivo. Cell 106: 685-696.

Malik, S. and Roeder, R.G. 2000. Transcriptional regulation through Mediator-like coactivators in yeast and metazoan cells. Trends Biochem. Sci. 25: 277-283.

Malik, S., Gu, W., Wu, W., Qin, J., and Roeder, R.G. 2000. The
USA-derived transcriptional coactivator PC2 is a submodule of TRAP/SMCC and acts synergistically with other PCs. Mol. Cell 5: 753-760.

Meisterernst, M., Roy, A.L., Lieu, H.M., and Roeder, R.G. 1991. Activation of class II gene transcription by regulatory factors is potentiated by a novel activity. Cell 66: 981-993.

Mittler, G., Kremmer, E., Timmers, H.T., and Meisterernst, M 2001. Novel critical role of a human Mediator complex for basal RNA polymerase II transcription. EMBO Rep. 2: 808813.

Myers, L.C. and Kornberg, R.D. 2000. Mediator of transcriptional regulation. Annu. Rev. Biochem. 69: 729-749.

Myers, L.C., Gustafsson, C.M., Hayashibara, K.C., Brown, P.O., and Kornberg, R.D. 1999. Mediator protein mutations that selectively abolish activated transcription. Proc. Natl. Acad. Sci. 96: 67-72.

Naar, A.M., Beaurang, P.A., Robinson, K.M., Oliner, J.D., Avizonis, D., Scheek, S., Zwicker, J., Kadonaga, J.T., and Tjian, R. 1998. Chromatin, TAFs, and a novel multiprotein coactivator are required for synergistic activation by $\mathrm{Sp} 1$ and SREBP-1a in vitro. Genes \& Dev. 12: 3020-3031.

Naar, A.M., Beaurang, P.A., Zhou, S., Abraham, S., Solomon, W., and Tjian, R. 1999. Composite co-activator ARC mediates chromatin-directed transcriptional activation. Nature 398: $828-832$

Nakajima, N., Horikoshi, M., and Roeder, R.G. 1988. Factors involved in specific transcription by mammalian RNA polymerase II: Purification, genetic specificity, and TATA boxpromoter interactions of TFIID. Mol. Cell. Biol. 8: 4028-4040.

Oelgeschlager, T., Tao, Y., Kang, Y.K., and Roeder, R.G. 1998 Transcription activation via enhanced preinitiation complex assembly in a human cell-free system lacking TAFIIs. Mol. Cell 1: 925-931.

Ozer, J., Moore, P.A., Bolden, A.H., Lee, A., Rosen, C.A., and Lieberman, P.M. 1994. Molecular cloning of the small (gamma) subunit of human TFIIA reveals functions critical for activated transcription. Genes \& Dev. 8: 2324-2335.

Pazin, M.J., Kamakaka, R.T., and Kadonaga, J.T. 1994. ATPdependent nucleosome reconfiguration and transcriptional activation from preassembled chromatin templates. Science 266: $2007-2011$

Ptashne, M. and Gann, A. 1997. Transcriptional activation by recruitment. Nature 386: $569-577$.

Rachez, C. and Freedman, L.P. 2001. Mediator complexes and transcription. Curr. Opin. Cell Biol. 13: 274-280.

Rachez, C., Suldan, Z., Ward, J., Chang, C.P., Burakov, D., Erdjument-Bromage, H., Tempst, P., and Freedman, L.P. 1998. A novel protein complex that interacts with the vitamin D3 receptor in a ligand-dependent manner and enhances VDR transactivation in a cell-free system. Genes \& Dev. 12: $1787-1800$.

Rachez, C., Lemon, B.D., Suldan, Z., Bromleigh, V., Gamble, M., Naar, A.M., Erdjument-Bromage, H., Tempst, P., and Freedman, L.P. 1999. Ligand-dependent transcription activation by nuclear receptors requires the DRIP complex. Nature 398: 824-828

Rachez, C., Gamble, M., Chang, C.P., Atkins, G.B., Lazar, M.A. and Freedman, L.P. 2000. The DRIP complex and SRC-1/ p160 coactivators share similar nuclear receptor binding determinants but constitute functionally distinct complexes. Mol. Cell. Biol. 20: 2718-2726.

Ranish, J.A., Yudkovsky, N., and Hahn, S. 1999. Intermediates in formation and activity of the RNA polymerase II preinitiation complex: Holoenzyme recruitment and a postrecruitment role for the TATA box and TFIIB. Genes \& Dev. 13: 49-63. 
Ruppert, S., Wang, E.H., and Tjian, R. 1993. Cloning and expression of human TAFII250: A TBP-associated factor implicated in cell-cycle regulation. Nature 362: 175-179.

Ryu, S., Zhou, S., Ladurner, A.G., and Tjian, R. 1999. The transcriptional cofactor complex CRSP is required for activity of the enhancer-binding protein Sp1. Nature 397: 446-450.

Sadowski, I., Ma, J., Triezenberg, S., and Ptashne, M. 1988. GAL4-VP16 is an unusually potent transcriptional activator. Nature 335: 563-564.

Sauer, F., Hansen, S.K., and Tjian, R. 1995. Multiple TAFIIs directing synergistic activation of transcription. Science 270: $1783-1788$

Sawadogo, M. and Roeder, R.G. 1985. Interaction of a genespecific transcription factor with the adenovirus major late promoter upstream of the TATA box region. Cell 43: 165175 .

Shykind, B.M., Kim, J., Stewart, L., Champoux, J.J., and Sharp, P.A. 1997. Topoisomerase I enhances TFIID-TFIIA complex assembly during activation of transcription. Genes \& Dev. 11: 397-407.

Sun, X., Zhang, Y., Cho, H., Rickert, P., Lees, E., Lane, W., and Reinberg, D. 1998. NAT, a human complex containing Srb polypeptides that functions as a negative regulator of activated transcription. Mol. Cell 2: 213-222.

Taatjes, D.J., Naar, A.M., Andel III, F., Nogales, E., and Tjian, R. 2002. Structure, function, and activator-induced conformations of the CRSP coactivator. Science 295: 1058-1062.

Tantin, D., Chi, T., Hori, R., Pyo, S., and Carey, M. 1996. Biochemical mechanism of transcriptional activation by GAL4VP16. Methods Enzymol. 274: 133-149.

Thompson, C.M., Koleske, A.J., Chao, D.M., and Young, R.A. 1993. A multisubunit complex associated with the RNA polymerase II CTD and TATA-binding protein in yeast. Cell 73: 1361-1375.

Van Dyke, M.W., Sawadogo, M., and Roeder, R.G. 1989. Stability of transcription complexes on class II genes. Mol. Cell. Biol. 9: 342-344.

Walker, S.S., Reese, J.C., Apone, L.M., and Green, M.R. 1996. Transcription activation in cells lacking TAFIIS. Nature 383: $185-188$.

Wang, W., Carey, M., and Gralla, J.D. 1992a. Polymerase II promoter activation: Closed complex formation and ATPdriven start site opening. Science 255: 450-453.

Wang, W., Gralla, J.D., and Carey, M. 1992b. The acidic activator GAL4-AH can stimulate polymerase II transcription by promoting assembly of a closed complex requiring TFIID and TFIIA. Genes \& Dev. 6: 1716-1727.

Woychik, N.A. and Hampsey, M. 2002. The RNA Polymerase II machinery. Structure illuminates function. Cell 108: 453463.

Yie, J., Senger, K., and Thanos, D. 1999. Mechanism by which the IFN-beta enhanceosome activates transcription. Proc. Natl. Acad. Sci. 96: 13108-13113.

Yuan, C.X., Ito, M., Fondell, J.D., Fu, Z.Y., and Roeder, R.G. 1998. The TRAP220 component of a thyroid hormone receptor- associated protein (TRAP) coactivator complex interacts directly with nuclear receptors in a ligand-dependent fashion. Proc. Natl. Acad. Sci. 95: 7939-7944.

Yudkovsky, N., Ranish, J.A., and Hahn, S. 2000. A transcription reinitiation intermediate that is stabilized by activator. $\mathrm{Na}$ ture 408: 225-229.

Zhou, Q., Lieberman, P.M., Boyer, T.G., and Berk, A.J. 1992. Holo-TFIID supports transcriptional stimulation by diverse activators and from a TATA-less promoter. Genes \& Dev. 6: $1964-1974$. 


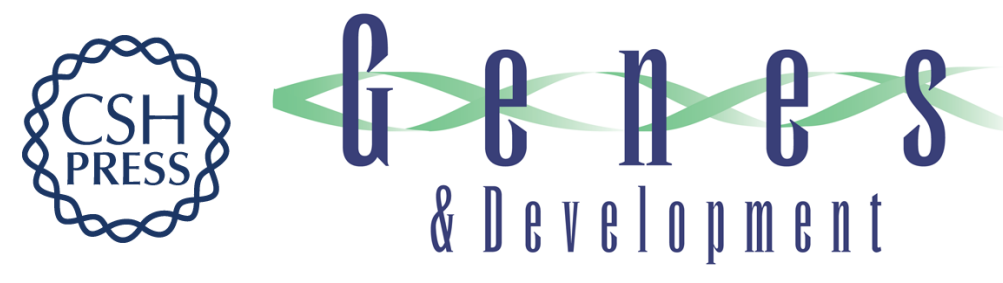

\section{TFIID and human mediator coactivator complexes assemble cooperatively on promoter DNA}

Kristina M. Johnson, Jin Wang, Andrea Smallwood, et al.

Genes Dev. 2002, 16:

Access the most recent version at doi:10.1101/gad.995702

References This article cites 75 articles, 31 of which can be accessed free at: http://genesdev.cshlp.org/content/16/14/1852.full.html\#ref-list-1

License

Email Alerting

Receive free email alerts when new articles cite this article - sign up in the box at the top Service right corner of the article or click here.

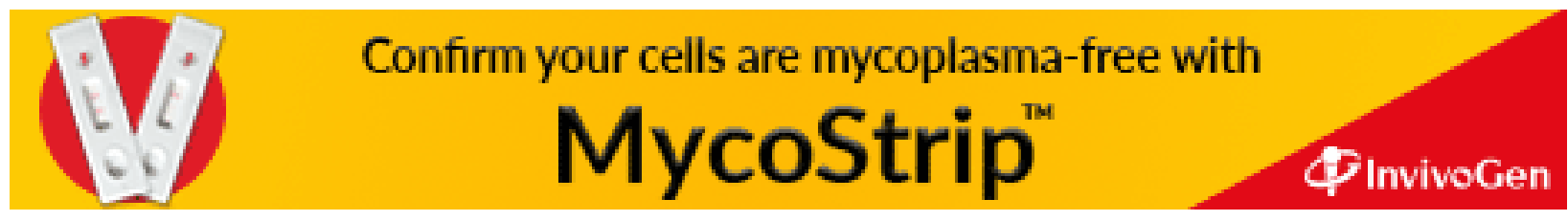

